\title{
Phosphorylation Process
}

National Cancer Institute

\section{Source}

National Cancer Institute. Phosphorylation Process. NCI Thesaurus. Code C16983.

The creation of a phosphate derivative of an org anic molecule. This is usually achieved by transferring a phosphate group from ATP via the action of a kinase. 